White PAPER FOR Planetary AND ASTROBIOLOGy SCIENCE STRATEgy

\title{
The Importance of Exploring Neptune's Aurora and Ionosphere
}

Principle author: Tom Stallard, University of Leicester, Tel: +44 116252 3589, e-mail: tss8@leicester.ac.uk

Co-authors and endorsers: Nahid Chowdhury ${ }^{1}$, John Clarke ${ }^{2}$, Ian Cohen ${ }^{3}$, Frank Crary ${ }^{4}$, Henrik Melin $^{1}$, Steve Miller ${ }^{5}$, Karl Mitchell ${ }^{6}$, Luke Moore², James O’Donoghue ${ }^{7}$, Abi Rymer ${ }^{3}$, Emma Thomas $^{1}$, Ron Vervack ${ }^{3}$

${ }^{1}$ University of Leicester, ${ }^{2}$ Boston University ${ }^{3}$ Johns Hopkins APL, ${ }^{4}$ LASP Boulder, ${ }^{5}$ University College London, ${ }^{6}$ Jet Propulsion Laboratory, , ${ }^{7}$ Japan Aerospace Exploration Agency 
Neptune's aurora are some of the least well understood within the entire solar system. The bright and complex aurora of Jupiter and Saturn have helped to reveal the vast current systems powered by the interchange of momentum between the immense underlying atmosphere and the enormous regions of magnetospheric plasma surrounding the planet. These drive a complex array of currents and chemistry into a very narrow and highly dynamic layer of the atmosphere, resulting in a wide variety of different emissions. Our observations of Ice Giant planets are far more limited, but current measurements suggest that auroral interactions are complicated by the highly complex magnetic fields that these worlds produce. Neptune may be unique in combining these, resulting in an aurora that is driven from the atmosphere, driving emissions through multiple auroral regions across the planet's disk, into a magnetosphere dominated by material from the moon Triton. Measurements of Neptune's aurora will greatly improve our understanding of the aurorae themselves, along with their atmospheric and magnetospheric context, and in turn provide a new understanding of all Ice Giant aurorae, both within the solar system and around other stars. This is particularly applicable to the large number of Neptune or mini-Neptune size exoplanets that are being discovered.

\section{Past observations of Neptune's aurora}

Our understanding of Neptune's aurora is limited almost entirely to radio, UV and in-situ observations made by Voyager 2.

Neptune produced a complex array of radio emissions, including the most intense, impulsive and narrow-banded emissions ever observed at a radio planet (Zarka et al., 1995), shown in Figure 1. These auroral emissions were positioned broadly over the magnetic poles, alongside smooth radio emissions that are unique to Uranus and Neptune. In addition, high-frequency smooth kilometric radio emissions were detected at Neptune's magnetic equator, and may have resulted from the complex magnetic structures in that region (Rabl et al., 1992).

Voyager 2 also detected extended ultraviolet $\mathrm{H}_{2}$ band emissions within Neptune's nightside. This night time airglow was observed across the disk, and was proposed to be powered by photoelectrons flowing along magnetically conjugate field lines that closed in the dayside. A brighter 10 Rayleigh emission was positioned around the southern magnetic pole, and was tentatively identified as a faint southern aurora (Broadfoot et al., 1989). However, since Voyager 2, despite several observations using the Hubble Space Telescope, Neptune's UV auroral emission remains undetected from Earth.

Voyager 2 did not include any near-infrared instrument and, despite decades of observations from Earth-based telescopes, the molecular ion $\mathrm{H}_{3}{ }^{+}$has never been observed at Neptune. This contrasts strongly against the wealth of detailed $\mathrm{H}_{3}{ }^{+}$measurements from the aurora and ionospheres of Jupiter, Saturn and Uranus. The most recent Neptune observations continued to show zero $\mathrm{H}_{3}{ }^{+}$emission (Melin et al., 2018), while resolving the continuum of reflected sunlight (despite the strong absorption from the methane rich lower atmosphere). This lack of emission means that $\mathrm{H}_{3}{ }^{+}$is less than 10 times as bright than predicted by atmospheric modelling, with this short fall in brightness explained though either a significantly cooler thermospheric temperature since Voyager, or due to chemical alteration from infalling neutral or plasma from the surrounding space environment (Moore et al., 2020). 


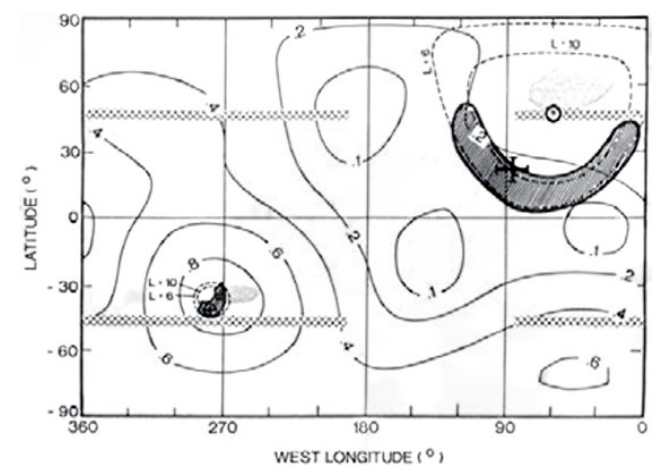

Figure 1: The predicted location of aurora at Neptune. On the left are planetary footprints of observed Neptune Kilometric Radiation with iso-contours of magnetic field amplitude (in Gauss) using the $\mathrm{O} 8$ magnetic field models taken from Zarka et al., 1993. On the right is the dip-angle from the 08 model, with the auroral region ( $70-75^{\circ}$ latitude, red), which are the likely locus for $\mathrm{UV}$, visible and $\mathrm{H}_{3}{ }^{+}$emissions and the region mapping to the rings (50-55 latitude, blue), which is the likely location of $\mathrm{HCO}^{+}$generation and $\mathrm{H}_{3}{ }^{+}$destruction. Similar production and destruction might also occur at Neptune's rotational equator, as is seen at Saturn.

\section{The planetary context for Neptune's aurora}

Although Uranus and Neptune both have highly non-dipolar magnetic fields, it is likely that the auroral coupling between the atmospheres and magnetospheres of these two worlds will have very different interactions.

The atmosphere of Uranus is highly extended, with limited vertical mixing due to the very low thermal output from the planet. As a result, it may be more difficult for the underlying atmosphere to drive momentum into the upper atmosphere, weakening the driving force of the thermosphere on the surrounding ions. Another unique feature of Uranus is the extreme tilt of the planet on its spin axis. In this orientation, the magnetic poles of Uranus will at times be "typical", closer to orthogonal to the solar wind with at other planets in the Solar System. In other seasons the magnetic poles will point toward and away from the Sun as the planet spins, with a daily cycle of flux tubes that are open then closed to the solar wind. Uranus may have "auroral seasons" when the magnetosphere is filled or empty and the auroral activity is much stronger or weaker.

A unique feature of Neptune is its significant source of plasma: Neptune's magnetosphere was observed to be full of heavy ions (Belcher et al., 1989; Mauk et al., 1995). These are possibly nitrogen coming from Triton - suggesting there might be a Triton neutral torus of average density comparable to or greater than Titan at Saturn (Cheng, 1990), but importantly, produced much closer to the planet, making the torus more akin to that of Io at Jupiter and Enceladus at Saturn.

The aurora at Uranus may be more Earth-like, dominated by solar wind interactions imposed from above; this could explain the strongly enhanced emissions observed on the nightside of Uranus, both in radio and UV. In contrast with this, radio emission at Neptune does not have this nightside bias, and Voyager did not observe the expected enhancement of alpha particles relative to protons, as one would expect for a solar wind driven system. As such, it seems likely that 
Neptune's auroral emission may be driven by the balance of momentum between ions accelerated by the neutral atmosphere and magnetospheric plasma, as occurs at both Jupiter and Saturn. Gas Giant aurora are dominated by this atmosphere-magnetosphere coupling, which sets up significant currents at the magnetic mapping of moons and at the break down in co-rotation, suggesting Neptune may have extended arcs of auroral emission, and may also include an auroral footprint at the magnetically mapped location of Triton.

This transfer of momentum between the underlying atmosphere and the surrounding space environment may result in a rich array of potential interactions that have recently been observed at Jupiter and Saturn. Within the auroral region, strong localized flows within the neutral atmosphere are likely to drive auroral enhancements in Saturn's aurora, which are the ultimate cause of Saturn's variable rotation rate (Smith, 2011). Within the equatorial regions, Jupiter has strong evidence of localized coupling between the Great Red Spot and the ionosphere, caused by acoustic waves transferring heating into the thermosphere (O’Donoghue et al., 2011), and has an ionospheric darkening at the magnetic equator, most probably driven by the relative flow of ions and neutrals, similar to the equatorial fountain observed at Earth (Stallard et al., 2018). At Saturn, there is a large current system near the equator that may be caused by the strong equatorial winds in the lower atmosphere which in turn drive currents in the ionosphere (Khurana et al., 2018). All these Gas Giant processes are likely to occur at Neptune, but the induced currents caused by the offset between the magnetic and rotational poles are likely to result in vastly more complex interactions.

At Saturn, there is also significant interactions between the ionosphere of the planet and its rings, with both ions and neutral particles from Saturn's rings falling into the atmosphere and greatly affecting the ionospheric composition. A similar process of infalling material may also occur at Neptune, and has been evoked as one possible reason why $\mathrm{H}_{3}{ }^{+}$has not yet been detected, with infalling $\mathrm{CO}$ from the rings potentially reducing the $\mathrm{H}_{3}{ }^{+}$density by an order of magnitude (Moore et al., 2020). However, as at Saturn, this results in a compositional change in the ionosphere, with $\mathrm{HCO}^{+}$becoming a dominant ion. Infrared observations of emissions from $\mathrm{HCO}^{+}$ may reveal the location of regions where $\mathrm{H}_{3}{ }^{+}$has been destroyed - and if this includes infalling ions, could light up the magnetic mapping of the rings on the planet. The predicted locations of $\mathrm{H} 3+$ and $\mathrm{HCO}+$ are highlighted in Figure 1.

Observing both auroral and ionospheric emissions at Neptune will thus provide a wealth of information about the extent of coupling between the magnetosphere and atmosphere of the planet. But the location of these emissions can also provide unique insights into the magnetic field structure at the surface of the planet, and thus inform our understanding of the planet's interior. At Jupiter, our understanding of the magnetic field is now informed by Juno's high resolution polar orbit around the planet (Connerney et al., 2018), but auroral emissions have previously been used to refine the magnetic model (Connerney et al., 1998) and to reveal the existence of a localized enhancement near the surface (Grodent et al., 2008) and more recently, the ionospheric equatorial darkening was shown to map out the magnetic equator exactly (Stallard et al., 2018).

Indeed, with only a single flyby of the planet and without a surface constraint for the magnetic field model of Neptune, the surface magnetic field is very poorly constrained, since, as 
Connerney (1993) states the "model neglects all contributions of degree 4 and above. Even some of the lower-degree model coefficients are likely to be inaccurate. In reality the surface field is likely to be very different from that portrayed in these figures, particularly in the weak field regions.” Despite this inherent inaccuracy, the magnetic configuration that Connerney (1993) modelled is incredibly enticing. The highly unique surface field structure includes the only planetary magnetic field within the solar system with four magnetic poles, as well as two magnetic equators (comparable with the two magnetic equators of Jupiter). These could result in four separate auroral regions, two northern and two southern, producing an auroral interaction unlike any seen in the solar system, shown in Figure 1. Exploring and understanding these unique and complex aurorae must be seen as a high-priority target for future observations.

Understanding the aurora of Neptune is also important in the broader context of future observations from the aurora of exoplanets. With the majority of planets currently detected classified as sub-Neptune sized worlds, understanding the magnetic field interactions at Neptune is essential to providing context for auroral observations from these worlds. In addition to potential radio aurora, recent modelling of the expected emission features within observational spectra from sub-Neptune worlds has shown that both ARIEL and JWST should be able to detect ionospheric $\mathrm{H}_{3}{ }^{+}$, along with $\mathrm{H}_{3} \mathrm{O}^{+}$(Bourgalais et al., 2020). These models also reveal ionospheres dominated by $\mathrm{HCO}^{+}$. These predictions highlight the great importance in better understanding the aurora at Neptune, in order to provide an ‘archetype’ for Ice Giant aurora around other stars.

\section{Future observations of Neptune's aurora and ionosphere}

Ongoing attempts to observe Neptune's aurora from Earth have failed, both in the infrared and ultraviolet. Future Earth-based telescopes are likely to continue to struggle to observe the aurora. The James Webb Space Telescope will provide a sensitivity of 100x that of Keck, which may allow the detection of $\mathrm{H}_{3}{ }^{+}$on the dayside of the planet, but the spatial resolution of JWST instruments are limited to $<30$ pixels across the disk, which will make it difficult to resolve any auroral morphology against the reflected sunlight from the complex underlying atmosphere. Future 30m+ telescopes may better resolve auroral features through a combination of high spatial and spectral resolution, but ultraviolet, visible and infrared auroral observations are all much more viable from the night side of Neptune. Radio emissions from Neptune are completely obscured by the Earth's ionosphere, requiring measurements from space, and for Voyager 2, radio observations could only be made five days before closest approach (Lamy, 2020).

It is thus essential to observe Neptune's aurora in close proximity, if we are to fully understand the rich auroral interactions at Neptune and to place observations of extra-solar planets in appropriate context. Here, we strongly advocate for a space mission to the Neptune system, including instrumentation that can measure both the magnetic environment around the planet, and well as auroral emissions from the planet itself. A range of potential instrumentation could provide the auroral observations needed, but the highest priorities are to separately measure the radio aurora alongside spatially resolved aurorae, using ultraviolet, visible or infrared emissions or, preferably a combination of all these. A flyby mission (e.g. the proposed Trident Discovery mission) could provide essential new information about the aurora, allowing us to greatly improve our understanding of the magnetospheric origins, revealing the extent of ring interactions and improving current magnetic field models using the auroral morphology. 
Alternatively, an orbiting spacecraft (e.g. Rymer et al., PMCS study 2020) would provide incredible depth to our understanding, just as Cassin has done at Saturn and Juno continues to do at Jupiter. Only with such space missions will we reveal the complexities of Neptune's aurora and the dynamics interactions that drive them.

\section{References}

Belcher, J. W., Bridge, H. S., Bagenal, F., Coppi, B., Divers, O., Eviatar, A., Gordon, G. S., Lazarus, A. J., McNutt, R. L., Ogilvie, K. W., Richardson, J. D., Siscoe, G. L., Sittler, E. C., Steinberg, J. T., Sullivan, J. D., Szabo, A., Villanueva, L., Vasyliunas, V. M., \& Zhang, M. (1989), Plasma Observations near Neptune: Initial Results from Voyager 2. Science, 246, 1478, doi: 10.1126/science.246.4936.1478

Bourgalais, J., Carrasco, N., Changeat, Q., Venot, O., Jovanović, L., Pernot, P., Tennyson, J., Chubb, K. L., Yurchenko, S. N., \& Tinetti, G. (2020), Ions in the Thermosphere of Exoplanets: Observable Constraints Revealed by Innovative Laboratory Experiments. The Astrophysical Journal, 895, 77, doi: 10.3847/1538-4357/ab8e2d

Broadfoot, A. L., Atreya, S. K., Bertaux, J. L., Blamont, J. E., Dessler, A. J., Donahue, T. M., Forrester, W. T., Hall, D. T., Herbert, F., Holberg, J. B., Hunten, D. M., Krasnopolsky, V. A., Linick, S., Lunine, J. I., Mcconnell, J. C., Moos, H. W., Sandel, B. R., Schneider, N. M., Shemansky, D. E., Smith, G. R., Strobel, D. F., \& Yelle, R. V. (1989), Ultraviolet Spectrometer Observations of Neptune and Triton. Science, 246, 1459, doi: 10.1126/science.246.4936.1459

Cheng, A. F. (1990), Triton torus and Neptune aurora. Geophysical Research Letters, 17, 1669, doi: 10.1029/GL017i010p01669

Connerney, J. E. P., Acuña, M. H., Ness, N. F., \& Satoh, T. (1998), New models of Jupiter's magnetic field constrained by the Io flux tube footprint. Journal of Geophysical Research, 103, 11929, doi: 10.1029/97JA03726

Connerney, J. E. P., Kotsiaros, S., Oliversen, R. J., Espley, J. R., Joergensen, J. L., Joergensen, P. S., Merayo, J. M. G., Herceg, M., Bloxham, J., Moore, K. M., Bolton, S. J., \& Levin, S. M. (2018), A New Model of Jupiter's Magnetic Field From Juno's First Nine Orbits. Geophysical Research Letters, 45, 2590, doi: 10.1002/2018GL077312

Grodent, D., Bonfond, B., Gérard, J.-C., Radioti, A., Gustin, J., Clarke, J. T., Nichols, J., \& Connerney, J. E. P. (2008), Auroral evidence of a localized magnetic anomaly in Jupiter's northern hemisphere. Journal of Geophysical Research (Space Physics), 113, A09201, doi: 10.1029/2008JA013185

Hunt, G. J., Provan, G., Bunce, E. J., Cowley, S. W. H., Dougherty, M. K., \& Southwood, D. J. (2018), Field-Aligned Currents in Saturn's Magnetosphere: Observations From the F-Ring Orbits. Journal of Geophysical Research (Space Physics), 123, 3806, doi: 10.1029/2017JA025067 
Khurana, K. K., Dougherty, M. K., Provan, G., Hunt, G. J., Kivelson, M. G., Cowley, S. W. H., Southwood, D. J., \& Russell, C. T. (2018), Discovery of Atmospheric-Wind-Driven Electric Currents in Saturn's Magnetosphere in the Gap Between Saturn and its Rings. Geophysical Research Letters, 45, 10,068, doi: 10.1029/2018GL078256

Lamy L. (2020) Auroral emissions from Uranus and Neptune. Phil. Trans. R. Soc. Lond. A, submitted

Mauk, B. H., Krimigis, S. M., Cheng, A. F., \& Selesnick, R. S. (1995), Energetic particles and hot plasmas of Neptune.. Neptune and Triton, 169

Melin, H., Fletcher, L. N., Stallard, T. S., Johnson, R. E., O'Donoghue, J., Moore, L., \& Donnelly, P. T. (2018), The quest for H3+ at Neptune: deep burn observations with NASA IRTF iSHELL. Monthly Notices of the Royal Astronomical Society, 474, 3714, doi: 10.1093/mnras/stx3029

Moore, L., Moses, J.I., Melin, H., Stallard, T.S., and O’Donoghue, J. Atmospheric implications of the lack of H3+ detection at Neptune. Phil. Trans. R. Soc. Lond. A, submitted

O'Donoghue, J., Moore, L., Stallard, T. S., \& Melin, H. (2016), Heating of Jupiter's upper atmosphere above the Great Red Spot. Nature, 536, 190, doi: 10.1038/nature18940

Rabl GKF et al. (1992) Neptune's smoothly varying radio emission between 600-800 kHz. Planet. Radio Emissions III, 309-316.

Smith, C. G. A. (2011), A Saturnian cam current system driven by asymmetric thermospheric heating. Monthly Notices of the Royal Astronomical Society, 410, 2315, doi: 10.1111/j.13652966.2010.17602.x

Stallard, T. S., Burrell, A. G., Melin, H., Fletcher, L. N., Miller, S., Moore, L., O'Donoghue, J., Connerney, J. E. P., Satoh, T., \& Johnson, R. E. (2018), Identification of Jupiter's magnetic equator through H3+ ionospheric emission. Nature Astronomy, 2, 773, doi: 10.1038/s41550-0180523-z

Zarka P et al. (1995) Radio emissions from Neptune. Neptune and Triton 74, 341-387. 